\title{
Tunable Berry curvature and transport crossover in topological Dirac semimetal KZnBi
}

\author{
Junseong Song $\mathbb{D D}^{1,2,6}$, Byung Cheol Park ${ }^{1,6}$, Kyung Ik Sim² ${ }^{2}$ Joonho Bang ${ }^{1}$, Sunghun Kim ${ }^{3}$, Zhuo Yang ${ }^{4}$, Yoshimitsu Kohama ${ }^{4}$, \\ Yeongkwan Kim ${ }^{3,5}$ and Sung Wng Kim (iD) ${ }^{1,2 凶}$
}

Topological Dirac semimetals have emerged as a platform to engineer Berry curvature with time-reversal symmetry breaking, which allows to access diverse quantum states in a single material system. It is of interest to realize such diversity in Dirac semimetals that provides insight on correlation between Berry curvature and quantum transport phenomena. Here, we report the transition between anomalous Hall and chiral fermion states in three-dimensional topological Dirac semimetal $\mathrm{KZnBi}$, which is demonstrated by tuning the direction and flux of Berry curvature. Angle-dependent magneto-transport measurements show that both anomalous Hall resistance and positive magnetoresistance are maximized at $0^{\circ}$ between net Berry curvature and rotational axis. We find that the unexpected crossover of anomalous Hall resistance and negative magnetoresistance suddenly occurs when the angle reaches to $\sim 70^{\circ}$, indicating that Berry curvature strongly correlates with quantum transports of Dirac and chiral fermions. It would be interesting to tune Berry curvature within other quantum phases such as topological superconductivity.

npj Quantum Materials (2021)6:77; https://doi.org/10.1038/s41535-021-00378-7

\section{INTRODUCTION}

Berry's phase $\left(\Phi_{B}\right)$ is a geometric quantum phase, which is manifested in the magnetic field (B)-induced cyclotron motion of fermions along closed loop in momentum $(k)$ space around a Dirac point ${ }^{1,2}$. In transport experiments, the $\Phi_{B}$ has been verified from the analysis of Shubnikov-de Haas $(\mathrm{SdH})$ oscillation, which gives a nonzero intercept of zeroth Landau level $(\mathrm{LL})^{3,4}$. The $\Phi_{\mathrm{B}}$ with a quantized $\pi$ value is defined by an integral of the Berry curvature $\left(\Omega_{k}\right)$ over the closed Fermi surface (FS) in k space, bearing a resemblance to an electric monopole of Gauss law ${ }^{1,2}$. This effective magnetic field of $\Omega_{k}$ is strongly correlated with a diversity of quantum transport phenomena, such as anomalous and quantum Hall effects ${ }^{5,6}$, chiral magnetic effect ${ }^{7,8}$, and topological superconductivity ${ }^{9}$. The nonzero $\Omega_{k}$ can be generated through either time-reversal and inversion symmetry breakings and the resulting $\Omega_{k}$ is locally distributed in the Brillouin zone ${ }^{1,2}$. Regardless of the magnetization of a material, the modulation of $\Omega_{\mathrm{k}}$, which can be achieved by manipulating symmetries and band structures, will dominate quantum transport properties of topological materials.

Three-dimensional topological Dirac semimetal (3D TDS) state can be a central hub to access diverse topological states with a distinct quantum transport phenomenon by lifting a Dirac degeneracy with external stimuli ${ }^{10}$. For instance, a topological phase transition (TPT) from the 3D TDS to Weyl semimetal occurs by time-reversal symmetry breaking under $B$, which splits a degenerate Dirac cone into two Weyl cones with opposite $\Omega_{\mathrm{k}}$ hotspots $^{10,11}$ (Fig. 1a). Interestingly, the axial splitting of 3D Dirac cones can be adjusted according to the direction of external $B$, allowing the tuning of the $\Omega_{k}$ with the incidence angle $(\theta)$ of $B$. This angular dependence of the $\Omega_{k}$ has been of interest in the TDSs such as $\mathrm{Na}_{3} \mathrm{Bi}$ (refs. ${ }^{8,11}$ ), $\mathrm{Cd}_{3} \mathrm{As}_{2}$ (refs. ${ }^{12,13}$ ), and $\mathrm{ZrTe}_{5}$ (refs. ${ }^{5,6,14,15}$ ) due to the possibility to show a variety of exotic quantum transport phenomena, such as the negative magnetoresistance (NMR) induced by chiral anomaly $7,8,13,14$ and anomalous Hall effect $(\mathrm{AHE})^{5,15}$ originate from the $\Omega_{\mathrm{k}}$. Beyond that, tuning the $\Omega_{\mathrm{k}}$ would be important for controlling the mixed and separated states of such quantum transport phenomena to understand the correlation between Dirac and Weyl fermions in the topological semimetals, as we found that the NMR and AHE occurred with a reversal behavior on the angle-dependent $\Omega_{\mathrm{k}}$ (Fig. 1b). For the precise tuning of the $\Omega_{k}$, it is necessary to exploit a 3D TDS material with a simple band structure having only two Dirac cones robustly protected by a crystal symmetry and a FS topology accommodating only a single monopole without disturbance from other bands.

Such a 3D TDS can be found in ABC compounds (A: alkali metal, B: transition metal, $C$ : chalcogen element) with a $P 6_{3}$ / $m m c$ group symmetry, whose crystal structure is constructed by an alternative stacking of honeycomb BC layers and A ion layers, guaranteeing the TDS state from the threefold rotational and inversion symmetries ${ }^{16}$ (Fig. 1c). Among them, KZnBi has been found as the 3D TDS with only two Dirac cones that are exclusively composed from $\mathrm{Zn}-4 s$ and $\mathrm{Bi}-6 p$ orbitals of the $\mathrm{ZnBi}$ honeycomb lattice ${ }^{17}$ (Fig. 1d), providing a platform to study the transport phenomena of Dirac quasiparticles. In contrast to Weyl semimetals that usually have a complex band structure with multiple $\Omega_{\mathrm{k}}$ hotspots ${ }^{2}$, the simple band structure of the $3 \mathrm{D}$ TDS KZnBi allows to resolve the $\Omega_{\mathrm{k}}$ distribution under $\mathrm{B}$ explicitly and to modulate the $\Omega_{\mathrm{k}}$ with a weak $B$ efficiently (Supplementary Fig. 1). Here, from the $\theta$-dependent magnetotransport measurements, we find that the tunable $\Omega_{k}$ determines the quantum transport characters of Dirac fermions (DFs) and chiral fermions (CFs), demonstrating an abrupt transition between anomalous Hall and CF states.

${ }^{1}$ Department of Energy Science, Sungkyunkwan University, Suwon 16419, Republic of Korea. ${ }^{2}$ Center for Integrated Nanostructure Physics, Institute for Basic Science, Suwon 16419, Republic of Korea. ${ }^{3}$ Department of Physics, Korea Advanced Institute of Science and Technology, Daejeon 34141, Republic of Korea. ${ }^{4}$ Institute for Solid State Physics, The University of Tokyo, Kashiwa, Chiba 277-8581, Japan. ${ }^{5}$ Graduate School of Nanoscience and Technology, Korea Advanced Institute of Science and Technology, Daejeon 34141 , Republic of Korea. ${ }^{6}$ These authors contributed equally: Junseong Song, Byung Cheol Park. ${ }^{{ }^{*}}$ email: kimsungwng@skku.edu 


\section{a}

Anomalous Hall state

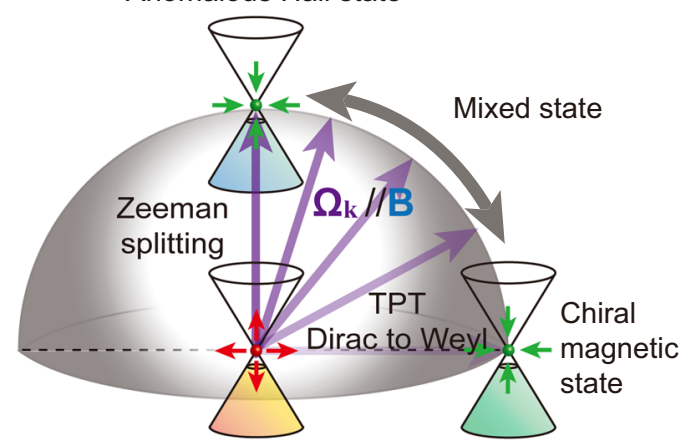

C

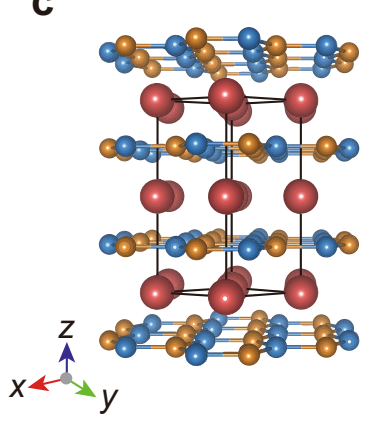

b

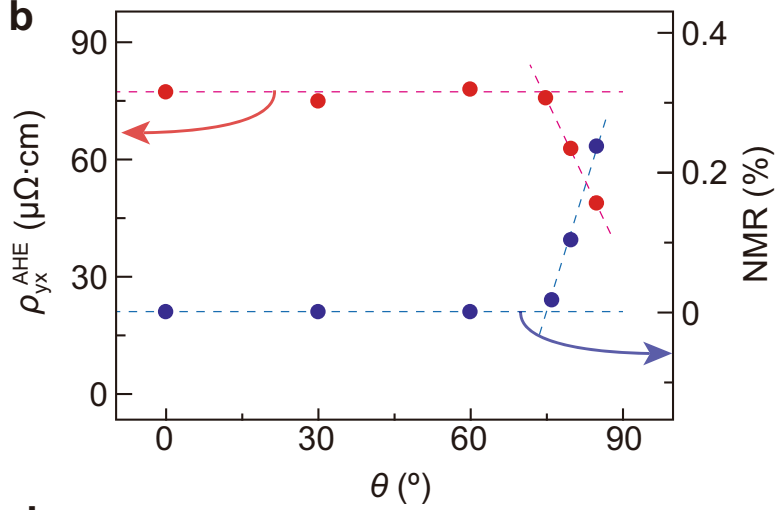

d

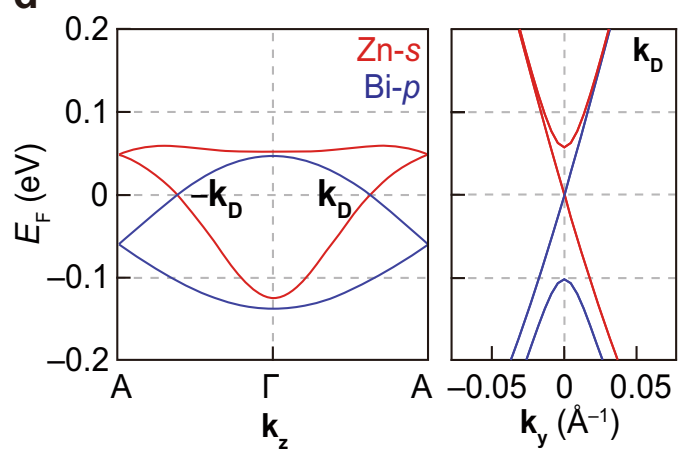

Fig. 1 Tunable Berry curvature and quantum transport phenomena in 3D TDS KZnBi. a B-dependent Weyl cone splitting and net $\Omega_{\mathrm{k}}$, which determines quantum transport phenomena of the 3D TDS. The source (red arrows) and sink (green arrows) of the $\Omega_{\mathrm{k}}$ are located at two Weyl points. The net $\Omega_{\mathrm{k}}$ (purple arrows) is tunable by varying the angle $(\theta)$ of external B. TPT is an abbreviation of topological phase transition. $\mathbf{b} \theta-$ dependent anomalous Hall resistivity ( $\rho_{\mathrm{yx}}^{\mathrm{AHE}}$, red dots) at saturation and negative magnetoresistance (NMR, blue dots). Dashed lines are guides to the eyes. c Crystal structure of the KZnBi constructed by planar ZnBi honeycomb and $\mathrm{K}$ ion layers (left panel), which has $C_{3 z}$ rotational and inversion symmetries (right panel). d Band structure of the KZnBi that contains two Dirac cones at $\pm k_{D}$ (left panel) which is constructed by $Z n-s$ (red lines) and Bi- $p$ (blue lines) orbitals (right panel).

\section{RESULTS}

\section{Quantum transport of DFs}

First, we examined the basic character of quantum transport of the DFs in the 3D TDS KZnBi sample. Figure 2a shows the metallic behavior in the $T$-dependent electrical resistivity $\left(\rho_{\mathrm{xx}}(T)\right)$ at $4 \mathrm{~K} \leq$ $T \leq 300 \mathrm{~K}$. In the MR measurements, we observed the unsaturated behavior and obtained the high value of $700 \%$ under $14 \mathrm{~T}$ at $4 \mathrm{~K}$ (Fig. 2b). It is noticeable that multiple SdH oscillations appear in the MR data in the range of $0 \leq B \leq 14 \mathrm{~T}$, implying a high mobility of the DFs as a characteristic transport feature of TDS materials $s^{8,12,13}$. These clear Dirac transports are ascribed to the planar $\mathrm{ZnBi}$ honeycomb layers responsible for the formation of 3D Dirac cones and to the high-quality crystallinity of single crystal $\mathrm{KZnBi}$ with a very low impurity level. Moreover, a simple Dirac band benefited from the planar ZnBi honeycomb layer (Fig. 1c, d) is also featured in the remarkably low magnitude of $B$ for the quantum limit. To investigate the transport behavior beyond the quantum limit, we performed tunnel diode oscillator (TDO) measurements. We presented the graph of $B$ versus $1 / \Delta F_{\mathrm{TDO}}$ where the inverse of frequency variation, $\Delta F_{\mathrm{TDO}}$, in the TDO data is proportional to the resistance in the transport data (Fig. 2c). A linear increase of the $1 / \Delta F_{\mathrm{TDO}}$ beyond quantum limit of $\sim 16 \mathrm{~T}$ (red dashed line in Fig. $2 \mathrm{C}$ ) implies a crossing of the zeroth $\operatorname{LL}(n=0)$ with the Fermi level $\left(E_{\mathrm{F}}\right)$ (refs. ${ }^{3,18,19}$ ).

Next, we characterized the 3D Dirac state of the KZnBi from the analysis on the $\mathrm{SdH}$ oscillations, which reflects the Landau quantization under $\mathrm{B}$. The longitudinal resistance $\left(R_{\mathrm{xx}}\right)$ component is given in the form of $\mathrm{d} R_{\mathrm{xx}} / \mathrm{dB}$ to show the $\mathrm{SdH}$ oscillations, revealing the oscillation frequency, $F=1 / d(1 / B)=4.69 \mathrm{~T}$ (Fig. $2 \mathrm{~d}$ ). Based on the Onsager relationship $F=(\hbar / 2 \pi e) S_{F}$, where $\hbar$ is reduced Plank's constant and e is elementary charge ${ }^{3}$, a small cross-sectional area $\left(S_{F}=0.00046 \AA^{-2}\right)$ of the FS is obtained. For a circular FS, a Fermi wavevector also shows a small value $\left(\mathrm{k}_{\mathrm{F}}=\right.$ $0.012 \AA^{-1}$ ) is extracted from the relation ${ }^{20}$ of $S_{F}=\pi k_{F}^{2}$, implying a simple band structure with sharp 3D Dirac cones in the KZnBi. The extremely small value of the effective mass $\left(m^{*}=0.012 m_{0}\right.$ where $m_{0}$ is electron mass) is obtained at $B=4.03 \mathrm{~T}$ (inset of Fig. $2 \mathrm{~d}$ ), by fitting the $T$-dependent oscillation amplitude to Lifshitz-Kosevich formula ${ }^{3}, \Delta \rho_{x x}(T, B) / \rho_{x x}(T=4 \mathrm{~K}, B)=\mathrm{e}^{-\Lambda_{D}}(\Lambda / \sinh (\Lambda))$, where $\Lambda=2 \pi^{2} k_{\mathrm{B}} T / \beta, \Lambda_{\mathrm{D}}=2 \pi^{2} k_{\mathrm{B}} T_{\mathrm{D}} / \beta$, and $\beta=\mathrm{ehB} / 2 \pi m^{*}$. Here, $k_{\mathrm{B}}$ is a Boltzmann constant and $T_{\mathrm{D}}$ is a Dingle temperature. From the Fermi velocity $\left(v_{\mathrm{F}}=\hbar \mathrm{k}_{\mathrm{F}} / \mathrm{m}^{*} \cong 1.19 \times 10^{6} \mathrm{~m} \cdot \mathrm{s}^{-1}\right)$, the $E_{\mathrm{F}}=\hbar v_{\mathrm{F}} \mathrm{k}_{\mathrm{F}} \cong$ $93.6 \mathrm{meV}$ below the Dirac node is calculated ${ }^{20}$. All values we extracted from the $\mathrm{SdH}$ oscillations are well matched with the previous report ${ }^{17}$. These physical parameters of the DFs in the $\mathrm{KZnBi}$ well describe the characteristic feature of the DFs (Supplementary Table 1 for all physical parameters).

\section{Identification of nontrivial $\Phi_{\mathrm{B}}$ from Landau fan diagram}

The 3D Dirac cones of the $\mathrm{KZnBi}$ accommodate a nontrivial $\Phi_{\mathrm{B}}$, which can be obtained from the analysis of the $\mathrm{SdH}$ oscillations. According to the Lifshitz-Onsager rule for the $\mathrm{SdH}$ analysis ${ }^{3}$, the $R_{\mathrm{xx}}$ is expressed by $R_{\mathrm{xx}}=R_{0}[1+A(\mathrm{~B}, T) \cos 2 \pi(F / \mathrm{B}+\gamma-\delta)]$, where $R_{0}$ is the background resistance, $A(\mathrm{~B}, T)$ is the amplitude of the $\mathrm{SdH}$ oscillation, and $\gamma$ is the values of $1 / 2-\Phi_{B} / 2 \pi$, which are determined by the value of $\Phi_{B}$, where it is close to 0 for nontrivial $\Phi_{\mathrm{B}}$ of $\pi$, and $\delta$ is the degree of two dimensionality of FS $\left(\delta=0\right.$ for $2 D$ and $\pm 1 / 8$ for $3 D$ ). In case of a nontrivial $\Phi_{B}$, the value of $|\gamma-\delta|=\left|1 / 2-\Phi_{B} / 2 \pi-\delta\right|$ located between 0 and $1 / 8$. For the identification of nontrivial $\Phi_{B}$, we displayed the LL fan 

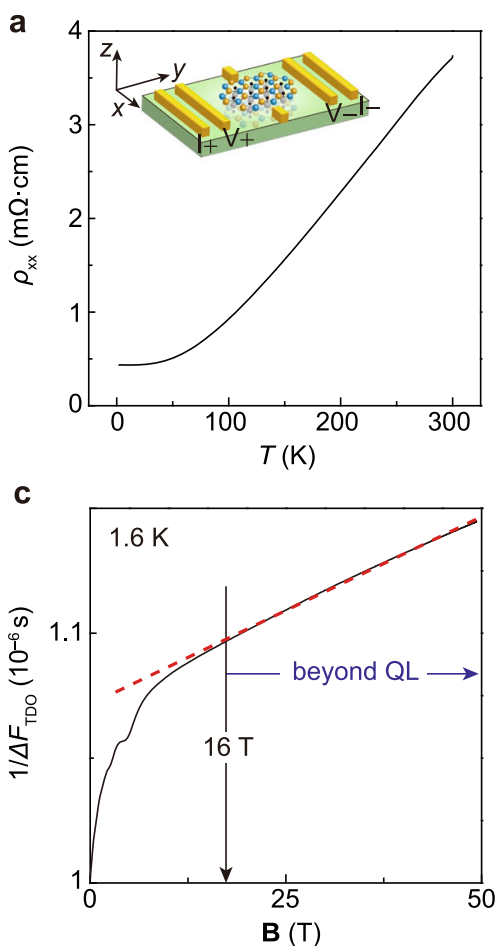

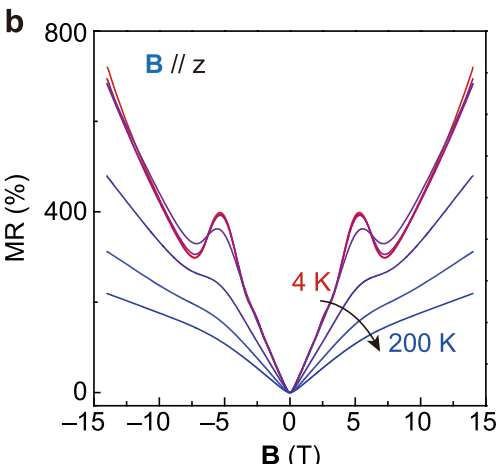

d

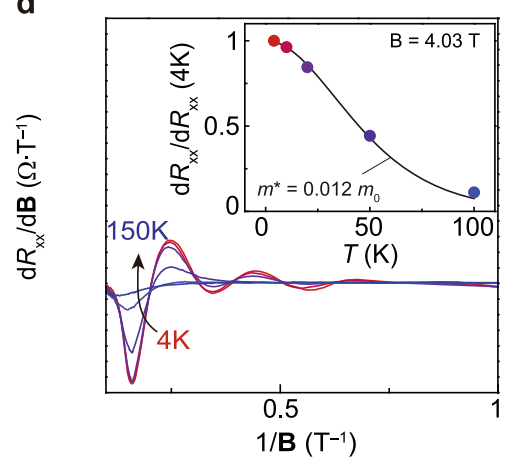

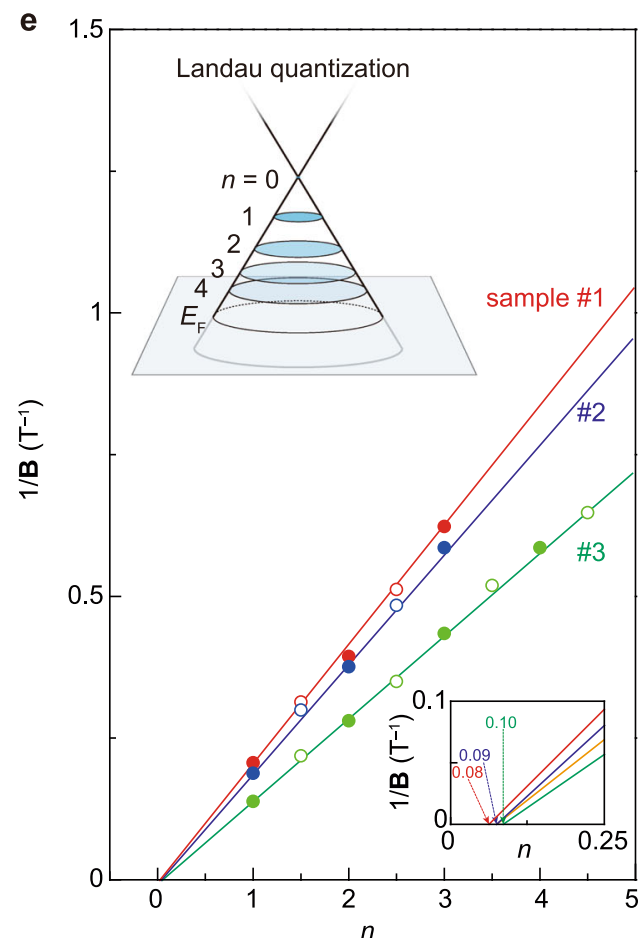

Fig. 2 Evidence of nontrivial Berry phase in 3D TDS KZnBi. a T-dependent $\rho_{\mathrm{xx}}$, which shows a metallic behavior of DFs. Illustration for transport measurements is shown in the inset. b MR data in the range of $-14 \mathrm{~T} \leq \mathrm{B} \leq 14 \mathrm{~T}$ at $4 \mathrm{~K} \leq T \leq 200 \mathrm{~K}$. $\mathbf{c}$ TDO data in the range of $0 \mathrm{~T} \leq$ $\mathrm{B} \leq 49 \mathrm{~T}$ at $1.6 \mathrm{~K}$. The linear MR (red dashed line) shows quantum limit behavior. $\mathbf{d ~ S d H}$ oscillations in $\mathrm{d} R_{\mathrm{xx}} / \mathrm{dB}$ at $4 \mathrm{~K} \leq T \leq 200 \mathrm{~K}$ (colored lines in the main panel) and T-dependent oscillation amplitudes (colored circles in the inset) at $\mathrm{B}=4.03 \mathrm{~T}$. $\mathrm{m}^{*} \cong 0.012 \mathrm{~m}_{0}$ is obtained from Lifshitz-Kosevich analysis (black line in the inset). e Landau fan diagram for three KZnBi samples (red, blue, and green for samples \#1, \#2 and \#3, respectively), which has integer indices (filled circles) and half-integer indices (open circles). Landau quantization with integer indices is described in the left top inset. The $x$-intercepts (right bottom inset) that are lying between 0 and $\pm 1 / 8$ imply a nontrivial $\Phi_{B}$.
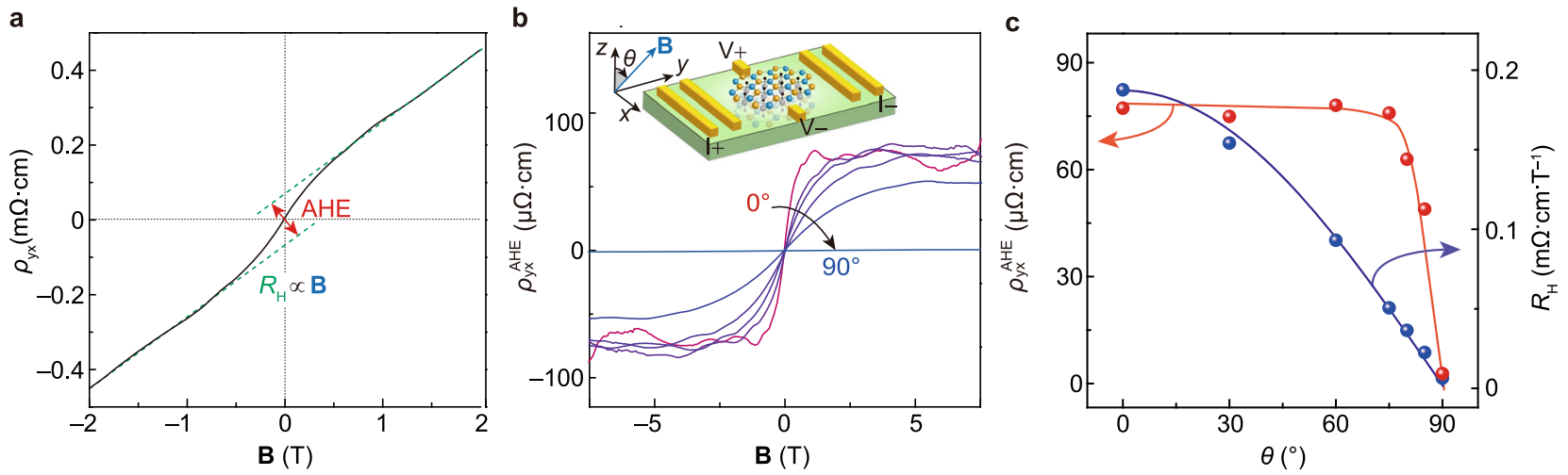

Fig. 3 Unusual angle-dependent anomalous Hall effect. a The $\rho_{\mathrm{yx}}$ in the range of $-2 \mathrm{~T} \leq \mathrm{B} \leq 2 \mathrm{~T}$ at $4 \mathrm{~K}$. A linear background (green dashed lines) is attributed to the ordinary Hall coefficient $\left(R_{\mathrm{H}}\right)$ and the anomaly near $\mathrm{B}=0$ (red two-headed arrow) is responsible for the $\rho_{\mathrm{yx}}^{\mathrm{AHE}}$. $\mathbf{b} \theta$ dependent $\rho_{\mathrm{yx}}^{\mathrm{AHE}}$ in the range of $0^{\circ} \leq \theta \leq 90^{\circ}$. Illustration of $\theta$-dependent Hall measurements is given in the inset. c Unusual $\theta$-dependent saturation of $\rho_{\mathrm{yx}}^{\mathrm{AHE}}$ (red dots and line) in contrast to conventional $\theta$-dependent $R_{\mathrm{H}}$ (blue dots) with a cosine profile (blue line).

diagram (Fig. 2e) by plotting the maxima (solid balls) and minima (open balls) of the SdH oscillations as integer and halfinteger indices, respectively. A linear extrapolation of the relation between $1 / B$ and $L L$ index gives the intercept value of $|\gamma-\delta|$ at zeroth LL. For the measured samples, the intercept values were in the range of the nontrivial $\Phi_{B}$ (inset of Fig. 2e, see Supplementary Figs. 2-4 for MR and SdH oscillations), implying the presence of the topologically nontrivial state in the KZnBi. Later, combined with AHE and NMR data, the state of the nontrivial $\Phi_{B}$ is revealed as the time-reversal-symmetry broken 3D TDS state.

\section{Tuning of $\boldsymbol{\Omega}_{\mathbf{k}}$ and $\boldsymbol{\theta}$-dependent AHE}

The $\Omega_{k}$ emerges and acts like an effective B in the 3D TDS when time-reversal symmetry is broken, giving net anomalous Hall current induced by the transverse velocity $\left(v_{A}=E \times \Omega_{k}\right)$ in the electrical field (E) (refs. ${ }^{5,15}$ ). To verify the AHE in the nonmagnetic 3D TDS KZnBi, we measured the Hall resistivity $\left(\rho_{\mathrm{yx}}\right)$ in the range of $-2 \mathrm{~T} \leq \mathrm{B} \leq 2 \mathrm{~T}$ at $4 \mathrm{~K}$ (see Supplementary Fig. 5 for the $\rho_{\mathrm{yx}}$ in the whole measured range of $-14 \mathrm{~T} \leq \mathrm{B} \leq 14 \mathrm{~T}$ ) as plotted in Fig. $3 \mathrm{a}$. It is clear that the $\rho_{\mathrm{yx}}$ shows the kink around zero $\mathrm{B}$, indicating the occurrence of AHE due to the $\Omega_{\mathrm{k}}$. We note that the easily 
distinguishable contribution of AHE (red arrow) indicates that the $\mathrm{KZnBi}$ crystal is in the clean limit where the quasiparticle lifetime is sufficiently long, as evidenced by the high mobility in the previous report (ref. $\left.{ }^{17}\right)$. The anomalous Hall resistivity $\left(\rho_{\mathrm{yx}}^{\mathrm{AHE}}\right)$ is separated from the total $\rho_{\mathrm{yx}}=\rho_{\mathrm{yx}}^{\mathrm{OHE}}+\rho_{\mathrm{yx}}^{\mathrm{AHE}}$, where $\rho_{\mathrm{yx}}^{\mathrm{OHE}}$ is the contribution of ordinary Hall effect (blue dotted line in the high $B$ region) extracted by a linear fitting ${ }^{5,15}$. The ordinary Hall coefficient $\left(R_{H}\right)$, which is obtained from the linear slope, indicates that the DFs of the present $\mathrm{KZnBi}$ are hole carriers with a density of $\sim 3.3 \times 10^{18}$ $\mathrm{cm}^{-3}$. This $p$-type carrier is in a good agreement with the recent results of angle-resolved photoemission spectroscopy and DFT calculation ${ }^{17}$. Although both Dirac band and small pocket are lying at the $E_{\mathrm{F}} \sim 100 \mathrm{meV}$, we showed that two-carrier model analysis cannot explain the nonlinearity of the Hall resistivity (Supplementary Fig. 6), ruling out the possibility of ordinary Hall effect. Also, the magnitude of $\rho_{y x}^{\mathrm{AHE}}$ for all measured samples shows the $E_{\mathrm{F}}$ independent value of $\sim 80 \mu \Omega \cdot \mathrm{cm}$, clarifying that the AHE was induced by $\Omega_{\mathrm{k}}$ (Supplementary Fig. 7). We note that the absence of the Zeeman splitting-induced separation in the SdH oscillation indicates the AHE does not originate from the spin-polarized massive DFs ${ }^{15}$.

Figure $3 \mathrm{~b}$ shows the separated $\rho_{\mathrm{yx}}^{\mathrm{AHE}}$, which has strong dependences on both the strength of $B$ and $\theta$ between $B$ and $z$ axis of sample. At the $\theta=0^{\circ}$, the $\rho_{\mathrm{yx}}^{\mathrm{AHE}}$ increases with $\mathrm{B}$ and saturates to the value of $\sim 78 \mu \Omega \cdot \mathrm{cm}$ at $4 \mathrm{~K}$ under $2 \mathrm{~T}$. The corresponding $\sigma_{\mathrm{yx}}^{\mathrm{AHE}} \approx 80 \Omega^{-1} \cdot \mathrm{cm}^{-1}$ of the $\mathrm{KZnBi}$ is a high value compared to that (typically $0.01-1 \Omega^{-1} \cdot \mathrm{cm}^{-1}$, ref. ${ }^{15}$ ) of the conventional materials with the AHE induced by extrinsic origins and it is comparable to that of topological semimetal, $\mathrm{ZrTe}_{5}$, with the AHE induced by nontrivial $\Phi_{\mathrm{B}}$ (Supplementary Fig. 5e). This phenomenon strongly indicates that the AHE of nonmagnetic $\mathrm{KZnBi}$ is intrinsically driven by the nonzero $\Omega_{\mathrm{k}}$. As illustrated in the Supplementary Fig. 1, the $\theta$-dependent $\Omega_{\mathrm{k}}$ can affect the transport properties of the AHE and reveal the correlation between $B$ and $\Omega_{\mathrm{k}}$. Indeed, a peculiar angular variation of the $\Omega_{\mathrm{k}}$ is uncovered from the $\theta$-dependent $\rho_{\mathrm{yx}}^{\mathrm{AHE}}$ curves at $4 \mathrm{~K}$ (Fig. 3b, c and Supplementary Fig. 5 for raw data). For each $\theta$-dependent $\rho_{\mathrm{yx}}^{\mathrm{AHE}}$ curve, the saturated $\rho_{\mathrm{yx}}^{\mathrm{AHE}}$ value appears at the different magnitude of $B$. The $\theta$-dependent curve of the $\rho_{\mathrm{yx}}^{\mathrm{AHE}}$ saturation values (red dots in Fig. $3 \mathrm{c}$ ) shows a sudden decrease from $\sim 70^{\circ}$ while the $\rho_{\mathrm{yx}}^{\mathrm{OHE}}(\theta)$ (blue dots in Fig. $3 c)$ smoothly follows a cosine profile of effective $B$ magnitude along the $z$ axis. This indicates that the critical crossover of $\rho_{\mathrm{yx}}^{\mathrm{AHE}}$ is irrelevant to the $\theta$-dependent change in the $B$ magnitude. This $\theta$-dependence of AHE resembles to that of Landau plot of other 3D TDS systems such as $\mathrm{Cd}_{3} \mathrm{As}_{2}$ (ref. ${ }^{12}$ ) and $\mathrm{ZrTe}_{5}$ (ref. ${ }^{21}$ ), where the origin was attributed to the TPT into a trivial phase. However, our study rules out such TPT scenario from the fact that the intercept value of zeroth $L L$ is in the characteristic range of nontrivial $\Phi_{\mathrm{B}}$ over the whole $\theta$ range (Supplementary Fig. 8). Alternatively, we suggest that a change in the FS topology plays a key role in modulating the $\Omega_{\mathrm{k}}$. At $\theta=0^{\circ}$, two Weyl cones, which are split along the $k_{z}$ direction, have a monopole in their own FSs, giving the nonzero net $\Omega_{\mathrm{k}}$. In contrast, over $\theta=70^{\circ}$, both the source and sink monopoles can be simultaneously enclosed by each $\mathrm{FS}^{22}$ due to the anisotropy of the FS of the $\mathrm{KZnBi}$ (Supplementary Fig. 4b). This $\theta$ dependent variation of the AHE verifies the modulation of the $\Omega_{k}$, which can stimulate a utilization of the ultrastrong $B$ (refs. ${ }^{23,24}$ ) for exploring exotic quantum phenomena inside the honeycomb layered TDS.

\section{$\boldsymbol{\theta}$-dependent evolution of NMR and its correlation with AHE}

Another representative quantum transport phenomenon in Dirac semimetals, the chiral anomaly-induced NMR, is also governed by the tuning of $\Omega_{\mathrm{k}}$. The NMR of chiral Weyl fermions in nonmagnetic $\mathrm{KZnBi}$ originates from a backscattering-free ballistic current of Weyl fermions driven by a chiral charge pumping $8,14,25,26$. While the measured MR shows positive values with prominent $\mathrm{SdH}$ oscillations under the condition of $\mathrm{B}$ perpendicular to current $\mathrm{I}\left(\theta=0^{\circ}\right)$, its magnitude significantly decreases as $\theta$ increases to $90^{\circ}$ (Fig. 4 a, see Supplementary Fig. 4 for FS analysis). We note that the sign of the MR remains positive up to $\theta=70^{\circ}$ but it abruptly turns to the negative value above $\theta=70^{\circ}$ (Fig. 4b). The NMR becomes larger when the $\theta$ is greater than $70^{\circ}$ and maximized at $90^{\circ}$ (Fig. 4c), which can be explained by the chiral magnetic effect determined by a product of B and I (inset of Fig. 4c) $8,14,25,26$. The upturn in MR values after $\sim 0.25 \mathrm{~T}$ may have originated from positive MR components of the weak antilocalization effect of nonzero Berry curvature $^{13}$ and the linear band dispersion of zeroth $\mathrm{LL}^{3,18,19}$. This competition between the positive and negative MR components inevitably changes with the applied B, giving different values of total MR at the different B (Supplementary Table 2 for comparison with other Dirac and Weyl semimetals). Remarkably, the sudden NMR change occurs at $\sim 70^{\circ}$ where the anomalous Hall resistance also shows a clear anomaly (Fig. $3 \mathrm{c}$ ). It is surprising that the NMR is clearly observed although the chirality of Weyl fermions is ill defined over $70^{\circ}$ due to the sudden shrink of the net $\Omega_{\mathrm{k}}$. As already described in Fig. $1 \mathrm{~b}$, this
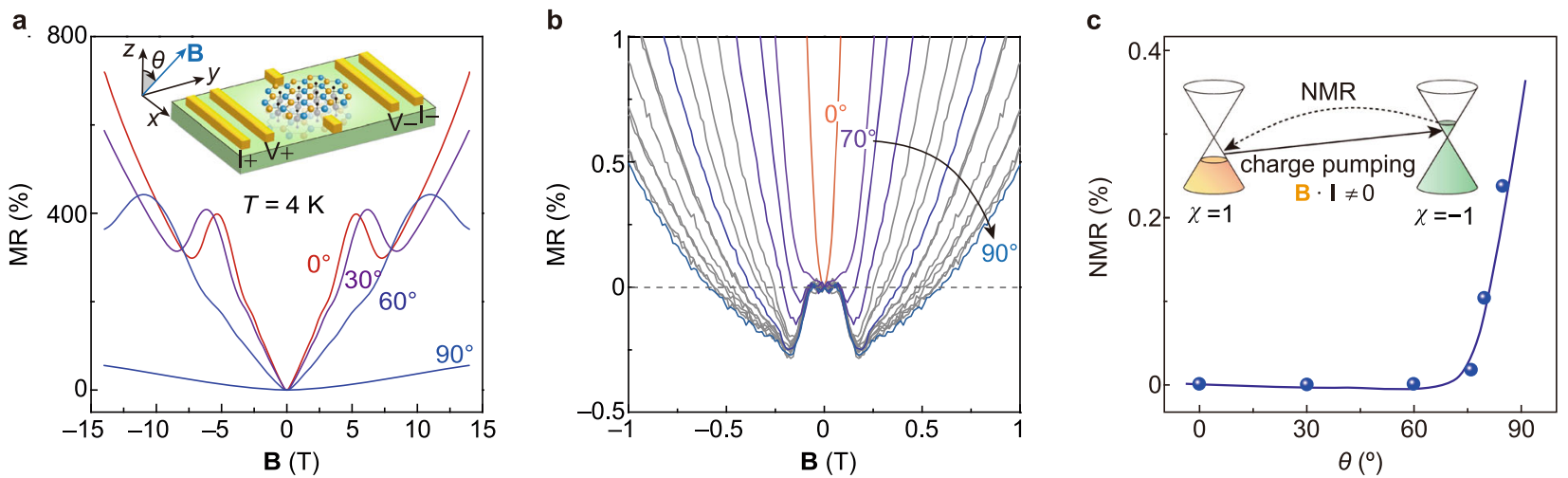

Fig. 4 Unusual angle-dependent chiral magnetic effect. a $\theta$-dependent $\mathrm{MR}$ for $\theta=0^{\circ}, 30^{\circ}, 60^{\circ}$, and $90^{\circ}$ at $T=4 \mathrm{~K}$, showing the maximal positive value of $\sim 700 \%\left(\theta=0^{\circ}, \mathrm{B}= \pm 14 \mathrm{~T}\right)$ with $\mathrm{SdH}$ oscillations. Illustration of the $\theta$-dependent MR measurements is given in the inset. b Magnified MR in the range of $-1 \mathrm{~T} \leq \mathrm{B} \leq 1 \mathrm{~T}$. NMR starts to appear at $70^{\circ}$ and its magnitude reaches the minimum of $\sim 0.25 \%$ at $90^{\circ}$. c Magnitudes of the NMR (blue dots and line) as a function of $\theta$, showing a crossover at $\sim 70^{\circ}$. The $\theta$ dependence of NMR is described by chiral magnetic effect (inset) in 3D TDS, which occurs under the collinear B and I. $X= \pm 1$ indicates chiralities of both Weyl cones. The chiral charge pumping induces a subsequent relaxation current, which is free from backscattering because of chirality protection and, therefore, is leading to a reduction of resistance. 
observation of the critical crossover between the anomalous Hall state and CF state in the 3D TDS indicates the experimental demonstration of their mixed and separated states. Because this intriguing crossover is revealed to be irrelevant to the TPT (Supplementary Fig. 8), its origin is needed to be studied further in the viewpoint of a hidden correlation between DFs and CFs.

\section{DISCUSSION}

In summary, we succeeded in identifying and modulating the $\Omega_{\mathrm{k}}$ in the 3D TDS KZnBi by varying the incidence $\theta$ of $B$. The nontrivial $\Phi_{B}$ was deduced from the nonzero intercept of LL fan diagram. Moreover, we observed the AHE and NMR in nonmagnetic 3D TDS, which are the intrinsic signature of nontrivial $\Phi_{\mathrm{B}}$ in the $\mathrm{KZnBi}$. It was suggested that the crossover between AHE and NMR can be explained with the FS topology without introducing the TPT. Our work provides a platform to study an interesting quantum transport entangled with the topological nature in the planar honeycomb layer structured 3D TDS, overcoming the difficulty in engineering the $\Omega_{\mathrm{k}}$ of the 2D honeycomb structured graphene.

\section{METHODS}

\section{Sample preparation}

The KZnBi single crystals are synthesized via self-flux method with the excess amount of $K$ metal to compensate the loss due to its evaporation ${ }^{17}$. The $\mathrm{K}: \mathrm{Zn}: \mathrm{Bi}$ mixture with a ratio of $1.3: 1: 1$ is placed in alumina crucible and then sealed under an evacuated quartz tube to avoid an oxidization during the reaction. The alumina crucible containing the mixture is place in a furnace and is heated to $630^{\circ} \mathrm{C}$ for $12 \mathrm{~h}$ and is cooled down to $300^{\circ} \mathrm{C}$ for $100 \mathrm{~h}$ and then to room $T$ by furnace quenching. High-quality copper ohmic contacts are formed on the sample by using silver conducting epoxy (inset of Fig. 2a) in a six-point probe configuration for measuring both longitudinal and transverse resistivities. This contact wiring process is carried out in glove box filled with a high-purity $\operatorname{Ar}(99.999 \%)$ gas to avoid the contact of the $\mathrm{KZnBi}$ from oxygen gas and moisture.

\section{Magneto-transport measurements}

To measure the transport properties of the $\mathrm{KZnBi}$ sample, physical property measurement system (PPMS Dynacool, Quantum Design) is used over the $T$ range from 4 to $300 \mathrm{~K}$. Transport behavior in extreme quantum limit is also investigated through TDO measurements on the $\mathrm{KZnBi}$ sample attached to the copper coil of the TDO circuit resonating at the frequency of $\sim 82 \mathrm{MHz}$. The sample with coil on the circuit is loaded to the chamber under a perpendicular $\mathrm{B}$ up to $49 \mathrm{~T}$ by using a nondestructive pulsed magnet at $T=1.6 \mathrm{~K}$. In all the $\theta$-dependent measurements, the rotator motor was calibrated with $\theta$-dependent resistance of the samples at $B$ of $14 T^{19,27-30}$.

\section{DATA AVAILABILITY}

The data that support the plots within this paper and other findings of this study are available from the corresponding author upon reasonable request.

Received: 1 April 2021; Accepted: 13 August 2021; Published online: 10 September 2021

\section{REFERENCES}

1. Xiao, D., Chang, M.-C. \& Niu, Q. Berry phase effects on electronic properties. Rev. Mod. Phys. 82, 1959-2007 (2010).

2. Yan, B. \& Felser, C. Topological materials: Weyl semimetals. Annu. Rev. Condens. Mater. Phys. 8, 337-354 (2017).

3. Murakawa, H. et al. Detection of Berry's phase in a bulk Rashba semicondcutor. Science 342, 1490-1493 (2013).

4. Zhang, Y., Tan, Y.-W., Stormer, H. L. \& Kim, P. Experimental observation of the quantum Hall effect and Berry's phase in graphene. Nature 438, 201-204 (2005).
5. Liang, T. et al. Anomalous Hall effect in $\mathrm{ZrTe}_{5}$. Nat. Phys. 14, 451-455 (2018).

6. Tang, F. D. et al. Three-dimensional quantum Hall effect and metal-insulator transition in $\mathrm{ZrTe}_{5}$. Nature 569, 537-541 (2019).

7. Parameswaran, S. A., Grover, T., Abanin, D. A., Pesin, D. A. \& Vishwanath, A Probing the chiral anomaly with nonlocal transport in three-dimensional topological semimetals. Phys. Rev. X 4, 031035 (2014).

8. Xiong, J. et al. Evidence for the chiral anomaly in the Dirac semimetal $\mathrm{Na}_{3} \mathrm{Bi}$. Science 350, 413-416 (2015).

9. Qi, X. L. \& Zhang, S. C. Topological insulators and superconductors. Rev. Mod. Phys. 83, 1057-1110 (2011).

10. Yang, B.-J. \& Nagaosa, N. Classification of stable three-dimensional Dirac semimetals with nontrivial topology. Nat. Commun. 5, 4898 (2014).

11. Wang, Z. et al. Dirac semimetal and topological phase transitions in $A_{3} B i(A=N a$, K, Rb). Phys. Rev. B 85, 195320 (2012).

12. Xiang, Z. J. et al. Angular-dependent phase factor of Shubnikov-de Haas oscillations in the Dirac semimetal $\mathrm{Cd}_{3} \mathrm{As}_{2}$. Phys. Rev. Lett. 115, 226401 (2015).

13. $\mathrm{Li}, \mathrm{H}$. et al. Negative magnetoresistance in Dirac semimetal $\mathrm{Cd}_{3} \mathrm{As}_{2}$. Nat. Commun. 7, 10301 (2016).

14. Li, Q. et al. Chiral magnetic effect in $\mathrm{ZrTe}_{5}$. Nat. Phys. 12, 550-554 (2016).

15. Sun, Z. et al. Large Zeeman splitting induced anomalous Hall effect in $\mathrm{ZrTe}_{5}$. npj Quantum Mater. 5, 36 (2020).

16. Zhang, X., Liu, Q., Xu, Q., Dai, X. \& Zunger, A. Topological insulators versus topological dirac semimetals in honeycomb compounds. J. Am. Chem. Soc. 140, 13687-13694 (2018).

17. Song, J. et al. Coexistence of surface superconducting and three-dimensional topological Dirac states in semimetal KZnBi. Phys. Rev. X 11, 021065 (2021).

18. Abrikosov, A. A. Quantum magnetoresistance. Phys. Rev. B 58, 2788-2794 (1998).

19. Zhao, Y. et al. Anisotropic Fermi surface and quantum limit transport in high mobility three-dimensional Dirac semimetal $\mathrm{Cd}_{3} \mathrm{As}_{2}$. Phys. Rev. X 5, 031037 (2015).

20. Ashcroft, N. W. \& Mermin, N. D. Solid State Physics (Brooks/Cole, 1976).

21. Liu, Y. et al. Zeeman splitting and dynamical mass generation in Dirac semimetal $\mathrm{ZrTe}_{5}$. Nat. Commun. 7, 12516 (2016).

22. Arnold, F. et al. Negative magnetoresistance without well-defined chirality in the Weyl semimetal TaP. Nat. Commun. 7, 11615 (2016).

23. Levy, N. et al. Strain-induced pseudo-magnetic fields greater than 300 Tesla in graphene nanobubbles. Science 329, 544 (2010).

24. Yan, W. et al. Strain and curvature induced evolution of electronic band structures in twisted graphene bilayer. Nat. Commun. 4, 2159 (2013).

25. Nielsen, H. B. \& Ninomiya, M. The Adler-Bell-Jackiw anomaly and Weyl fermions in a crystal. Phys. Lett. B 130, 389 (1983).

26. Son, D. T. \& Spivak, B. Z. Chiral anomaly and classical negative magnetoresistance of Weyl metals. Phys. Rev. B 88, 104412 (2013).

27. Huang, $X$. et al. Observation of the chiral-anomaly-induced negative magnetoresistance in 3D Weyl semimetal TaAs. Phys. Rev. X 5, 031023 (2015).

28. Zhang, C.-L. et al. Signatures of the Adler-Bell-Jackiw chiral anomaly in a Weyl fermion semimetal. Nat. Commun. 7, 10735 (2016).

29. Li, C.-Z. et al. Giant negative magnetoresistance induced by the chiral anomaly in individual $\mathrm{Cd}_{3} \mathrm{As}_{2}$ nanowires. Nat. Commun. 6, 10137 (2015).

30. Zhang, $C$. et al. Room-temperature chiral charge pumping in Dirac semimetals. Nat. Commun. 8, 13741 (2017).

\section{ACKNOWLEDGEMENTS}

This work was supported by a National Research Foundation of Korea (NRF) grant funded by the Korean government (Ministry of Science, ICT \& Future Planning) (No. 2015M3D1A1070639). B.C.P. acknowledges the support from the National Research Foundation of Korea (NRF-2019R1A6A3A01096112).

\section{AUTHOR CONTRIBUTIONS}

S.W.K. conceived the idea and organized the research. J.S. grew the single crystals and performed transport measurements. J.S., B.C.P., J.B., S.K., Y.Ki. and S.W.K. analyzed all experimental data. K.I.S. and B.C.P. performed theoretical calculations of Berry phase distribution. Z.Y. and Y.Ko. conducted high-field transport measurements with a tunnel diode oscillator. J.S., B.C.P. and S.W.K. wrote the manuscript with the input from all the authors.

\section{COMPETING INTERESTS}

The authors declare no competing interests. 


\section{ADDITIONAL INFORMATION}

Supplementary information The online version contains supplementary material available at https://doi.org/10.1038/s41535-021-00378-7.

Correspondence and requests for materials should be addressed to Sung Wng Kim.

Reprints and permission information is available at http://www.nature.com/ reprints

Publisher's note Springer Nature remains neutral with regard to jurisdictional claims in published maps and institutional affiliations.
Open Access This article is licensed under a Creative Commons Attribution 4.0 International License, which permits use, sharing, adaptation, distribution and reproduction in any medium or format, as long as you give appropriate credit to the original author(s) and the source, provide a link to the Creative Commons license, and indicate if changes were made. The images or other third party material in this article are included in the article's Creative Commons license, unless indicated otherwise in a credit line to the material. If material is not included in the article's Creative Commons license and your intended use is not permitted by statutory regulation or exceeds the permitted use, you will need to obtain permission directly from the copyright holder. To view a copy of this license, visit http://creativecommons. org/licenses/by/4.0/.

(c) The Author(s) 2021 